\title{
Autophagy Precedes Apoptosis in Angiotensin II-Induced Podocyte Injury
}

\author{
Su-Bin Seong ${ }^{\mathrm{a}}$ Dong-Soo Ha ${ }^{\mathrm{b}}$ Seo-Yun Min ${ }^{\mathrm{a}}$ Tae-Sun $\mathrm{Ha}^{\mathrm{a}, \mathrm{c}}$ \\ aDepartment of Pediatrics, College of Medicine, Chungbuk National University, Cheongju, Korea, \\ bDepartment of Biological Sciences, Korea Advanced Institute of Science and Technology (KAIST), \\ Daejeon, Korea, 'Department of Pediatrics, Chungbuk National University Hospital, Cheongju, Korea
}

\author{
Key Words \\ Angiotensin II • Podocyte • Autophagy $•$ Apoptosis $•$ PI3-kinase
}

\begin{abstract}
Background/Aims: Angiotensin II (Ang II) induces podocyte injury resulting in apoptosis in vitro and in vivo. However, the relationship between autophagy and apoptosis in Ang II-induced podocyte injury is unknown and the role of Ang II-induced autophagy in podocyte survival or death remains unclear. We investigated the sequential relationship between autophagy and apoptosis in Ang II-induced podocytes as well as the role of phosphatidylinositide 3-kinase (PI3-kinase). Methods: Mouse podocytes were incubated in media containing various concentrations of Ang II and at different incubation times. The changes of podocyte autophagy and apoptosis were observed by electron microscopy, confocal imaging, western blotting, and FACS assay according to the presence of Ang II. Results: Ang II enhanced the podocyte expression of the autophagic proteins, LC3A/B-II and beclin-1, and also increased the number of autophagosomes compared with control cells at early phase of 12 hours in a dose-dependent manner. This effect was inhibited by pretreatment with 3-methyladenine (3MA), a PI3-kinase class III inhibitor. Thereafter, the Ang II-induced enhancement in autophagy decreased, whereas, podocyte apoptosis appeared later at 24 hours in concentration- and time-dependent manners in FACS and TUNEL assays. 3-MA and LY294002, a pan PI3-kinase inhibitor, further increased Ang II-induced podocyte apoptosis. Suppression of autophagy by Atg5 siRNA could induce podocyte apoptosis and further augment high-dose Ang Il-induced podocyte apoptosis. Conclusion: These findings suggest that Ang II promotes autophagy in podocytes before apoptosis as an early adaptive cytoprotective mechanism for podocyte survival after Ang II treatment, and the transitional imbalance between autophagy and apoptosis causes podocyte injury.




\section{Cellular Physiology Cell Physiol Biochem 2019;53:747-759 \\ \begin{tabular}{ll|l} 
DOI: 10.33594/000000170 2019 The Author(s). Published by & C
\end{tabular} \\ and BiOChemistry Published online: 18 October 2019 Cell Physiol Biochem Press GmbH\&Co. KG \\ Seong et al.: Protective Role of Autophagy in Angiotensin II-Induced Podocyte \\ Apoptosis}

\section{Introduction}

Angiotensin II (Ang II) directly constricts vascular smooth muscle cells, stimulates aldosterone production, activates the sympathetic nervous system, and increases sodium reabsorption. These activities are mediated through Ang II type 1 receptor (AT1R) activation and contribute to the development of hypertension [1, 2]. In the kidney, Ang II mediates oxidative stress as well as apoptosis and inflammation via AT1R; stimulates transforming growth factor- $\beta 1$ production and signaling pathways in glomerular cells; and contributes to the development and maintenance of renal hypertrophy, extracellular matrix accumulation, proteinuria, and glomerulosclerosis [3-6].

Podocytes are terminally differentiated and highly specialized epithelial cells located on the outer surface of the glomerular basement membrane and play a crucial role in the regulation of glomerular filtration. Podocyte injury is an essential feature of proteinuria and progressive glomerular diseases [7-9]. Because the podocyte is a terminally differentiated cell, severe injury to the cell results in autophagy to remove unwanted or damaged proteins and organelles for its survival. However, if the insult is overwhelming, it may undergo apoptosis $[10,11]$.

Many stimuli can cause simultaneous apoptosis and autophagy. Ang II induces autophagy, which is further enhanced by rapamycin and inhibited by 3-methyladenine (3-MA), a PI3kinase class III inhibitor [12]. Ang II also induces apoptosis in podocytes by AT1R [13-15]. Ang II treatment in vitro and in vivo developed hypertension with vascular and podocyte contraction and proteinuria with increased podocyte permeability by a narrowing of the slit diaphragm, alterations in the distribution of nephrin, and increased podocyte apoptosis $[13,14]$. In the present study, we investigated our hypothesis that an imbalance between autophagy and apoptosis causes Ang II-induced podocyte injury.

\section{Materials and Methods}

Cell culture of mouse podocytes

Conditionally immortalized mouse podocytes were kindly provided by Peter Mundel (University of Harvard, Boston, MA, USA) and were cultured and differentiated as described previously [16]. In brief, to stimulate podocyte proliferation, cells were cultivated at $33^{\circ} \mathrm{C}$ (permissive conditions) in a culture medium supplemented with $10 \mathrm{U} / \mathrm{mL}$ mouse recombinant $\gamma$-interferon (Roche, Mannheim, Germany) to induce expression of temperature-sensitive large $\mathrm{T}$ antigen. To induce differentiation, podocytes were maintained at $37^{\circ} \mathrm{C}$ without $\gamma$-interferon (non-permissive conditions) for at least 2 weeks. We observed that gown podocytes were distributed evenly at all experimental conditions. All experiments were repeated at least three times.

Treatment conditions and preparation of antibodies

Mouse podocytes were incubated with various concentrations of Ang II (Sigma-Aldrich Inc., St. Louis, MO, USA) for 2, 6, 12, and 24 hours. Cells were exposed to $2 \mathrm{mM} \mathrm{3-MA} \mathrm{(Sigma-Aldrich} \mathrm{Inc.)} \mathrm{and} 5 \mu \mathrm{M}$ LY294002 (Cell Signaling Technology, Danvers, MA), a class III PI3-kinase and a pan PI3-kinase inhibitor, respectively. Cells were also treated by Chloroquine $(\mathrm{CQ}, 0.1 \mathrm{mM})$ to inhibit autolysosomal degradation and autophagy. The primary antibodies for anti-LC3A/B and anti-beclin-1 antibodies were purchased from Cell Signaling Technology (Beverly, MA, USA).

\section{Immunofluorescence staining}

Podocytes that were grown on type I collagen-coated glass cover slips incubated for 2, 6, 12, and 24 hours were fixed in $4 \%$ paraformaldehyde, permeabilized with $0.2 \%$ Triton X-100, blocked with $10 \%$ normal goat serum, and labeled with polyclonal rabbit anti-rat LC3A/B antibody (1:200, Cell Signaling Technology). Primary antibody-bound specimens were incubated with 1:200 (v/v) Alexa Fluor 488 for green and Alexa Fluor 594 for red (Invitrogen, Eugene, OR, USA)-conjugated secondary antibody at room temperature (RT) for 1 hour. Nuclei were stained with 4', 6-diamidino-2-phenylindole dihydrochloride (DAPI; Sigma). 


\section{Cellular Physiology Cell Physiol Biochem 2019;53:747-759 \\ \begin{tabular}{l|l|l}
\hline DOI: 10.33594/000000170 & (c)19 The Author(s). Published by
\end{tabular} \\ and Biochemistry Published online: 18 October 2019 Cell Physiol Biochem Press GmbH\&Co. KG \\ Seong et al.: Protective Role of Autophagy in Angiotensin II-Induced Podocyte \\ Apoptosis}

Coverslips were mounted in aqueous mountant and viewed with a Fluorescence microscope (TCS SP2 AOBS, Leica, Wetzlar, Germany). The percentage of podocytes showing accumulation of LC3 puncta (that is with at least five puncta per podocyte) was quantified. At least 200 cells were scored in each of three independent experiments.

\section{Western blotting}

The expression of autophagic proteins, including LC3A/B and beclin-1, were assayed by western blotting. The primary antibodies for anti-LC3A/B and anti-beclin-1 antibodies were diluted for western blotting as 1:1000 and 1:500, respectively. Total proteins from podocytes were extracted using protein extraction solution (PRO-PREP, Intron, Seongnam, Kyungki, Korea), containing leupeptin as a lysosomal inhibitor, according to the manufacturer's instructions. The confluently grown cell layers incubated with additives and extracted protein concentrations were determined. Thirty $\mu \mathrm{g}$ of boiled extracts was applied on 10 or $15 \%$ SDS-PAGE gels and transferred to polyvinylidene fluoride membranes (Bio-Rad Laboratories, Hercules, CA, USA). Then, the membranes were air-dried and blocked in $5 \%$ fat-free milk before incubation with the primary antibodies. After incubation with horseradish peroxidase-conjugated secondary antibodies (Santa Cruz Biotechnology), bands were detected using the enhanced chemiluminescence system (Amersham Biotech Ltd., Bucks, UK). In each experiment the ratio of absorbance of each molecule vs. $\beta$-tubulin was calculated and their density values were expressed as $\%$ of control (without Ang II).

\section{Transmission electron microscope (TEM) analysis}

Podocytes were washed and fixed in $2.5 \%$ glutaraldheyde (Sigma-Aldrich Inc,) for 3 hours, then, postfixed with $1 \%$ osmium tetroxide in buffer pH 7.4 at RT for 40 min, serially dehydrated with ethanol, and embedded in epoxy resin. Ultrathin sections were examined with a transmission electron microscope (Carl Zeiss, Libra 120, Oberkochen, Germany). The autophagosomes were labeled and measured using the ruler provided. The numbers of autophagic vacuoles were counted by two observers, and data were recorded.

\section{Terminal deoxynucleotidyl transferase dUTP nick end labeling (TUNEL) assay}

Podocytes that were grown on type I collagen-coated glass cover slips incubated for 2, 6, 12, and 24 hours were fixed in $4 \%$ paraformaldehyde for $20 \mathrm{~min}$, followed by permeabilization with $0.1 \%$ Triton X-100 for $10 \mathrm{~min}$ at RT. The TUNEL assay was performed according to the manufacturer's instructions (In Situ Cell Death Detection Kit, AP; Roche Molecular Biochemicals, Mannheim, Germany). After coverslips were mounted in mountant, the samples were immediately evaluated by a fluorescence microscope. The TUNEL index (apoptotic podocytes) was determined by counting the positively and negatively stained cells in each of 10 fields of vision.

\section{Fluorescence-activated cell sorting (FACS) assay}

Podocytes were washed twice, then, cells $\left(1 \times 10^{5} / \mathrm{mL}\right)$ were collected and suspended with 100 $\mu \mathrm{L}$ of $1 \times$ binding buffer (10 mM HEPES/NaOH pH 7.4, $140 \mathrm{mM} \mathrm{NaCl}, 2.5 \mathrm{mM} \mathrm{CaCl}_{2}$ ). Five $\mu \mathrm{L}$ of annexin V-fluorescein isothiocyanate (FITC) (BD Biosciences, San Jose, CA, USA) and $5 \mu \mathrm{L}$ of propidium iodide (PI) (BD Biosciences) were added for $15 \mathrm{~min}$ at RT in the dark. Thereafter, $400 \mu \mathrm{L}$ of $1 \times$ binding buffer was added and the percentages of apoptosis and necrosis in the adherent-cell fraction were determined by flow cytometry (FACSCalibur, BD Biosciences) after annexin V-FITC and PI labeling within 1 hour. All FACS analyses for apoptosis detection were performed in triplicate under each experimental condition.

\section{Small interference RNA (siRNA) for Atg5 transfection}

To investigate the function of Atg5 in Ang II-induced podocyte injury, siRNAs for Atg5 (Santa Cruz Biotechnology) was prepared using Lipofectamine. One day before transfection, the culture medium was removed and differentiated podocytes were cultivated in antibiotics-free RPMI 1640 medium supplemented with 10\% FBS. Cells were transfected with siRNA using Lipofectamine 2000 Transfection Reagent (Invitrogen, Carlsbad, CA, USA) according to the manufacturer's instructions. In brief, siRNA for Atg5, or control scrambled siRNA was diluted into each 6-well plate with Transfection Medium (Opti-MEM, Invitrogen) and incubated for $5 \mathrm{~min}$. In parallel, Lipofectamine was diluted with Transfection Medium (OptiMEM). Diluted Lipofectamine reagent and siRNA were mixed and incubated at room temperature for $20 \mathrm{~min}$. The medium was replaced with Opti-MEM and the siRNA/Lipofectamine mixture was added to the cells. 


\section{Cellular Physiology Cell Physiol Biochem 2019;53:747-759

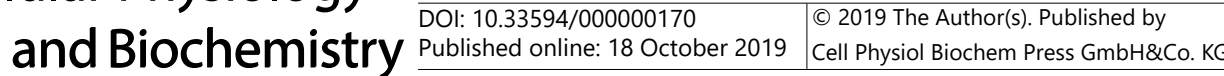

Seong et al.: Protective Role of Autophagy in Angiotensin II-Induced Podocyte

Apoptosis

After replacing the transfection mixture with RPMI 1640 medium supplemented with $10 \%$ FBS after $5 \mathrm{~h}$, the inhibitory effect of siRNA was confirmed by western blotting.

\section{Statistical analysis}

The results are expressed as mean values \pm standard deviation (SD). The statistical significance was assessed by nonparametric Kruskal-Wallis ANOVA analysis or Student's $t$-test using the SPSS 9.0.0 (SPSS, Chicago, IL) software program. $P$-values less than 0.05 were considered significant.

\section{Results}

Ang II induces beclin-1 expression of podocytes

Beclin-1 triggers rapid nucleation of its associated proteins in the phagophore at the initiation of autophagosome formation and the elongation of phagophore and the maturation of autophagosome is mediated by LC3-II; therefore, LC-3 and beclin-1 are two independent markers of autophagy. To determine the effect of Ang II on podocytes, we measured beclin 1 protein expression using western blotting. Podocytes were treated with graded concentrations of Ang II for various incubation periods. A high dose of Ang II $\left(10^{-6} \mathrm{M}\right)$ significantly increased beclin-1 protein expression at 6 hours and increased it further at 12 hours in a dose-dependent manner. Thereafter, beclin 1 protein expression decreased to below basal levels at 24 hours after correcting for $\beta$-tubulin levels $(n=$ 3) (Fig. 1A, B).

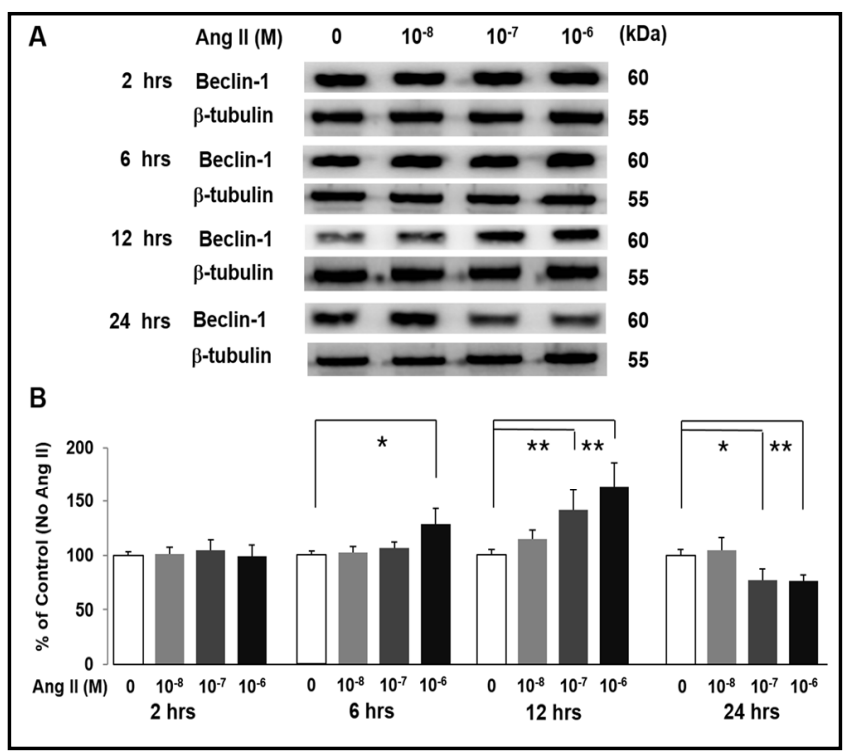

Fig. 1. Effects of Ang II on beclin-1 protein assayed using western blot. A high dose of Ang II $\left(10^{-6} \mathrm{M}\right)$ increases beclin-1 protein expression at 6 hours $(\mathrm{P}<0.05)$ and both $10^{-7} \mathrm{M}$ and $10^{-6} \mathrm{M}$ of Ang II further increases beclin-1 expression at 12 hours in a dose-dependent manner $(\mathrm{P}<0.01)$. Thereafter, beclin-1 protein expression decreases to below the basal level (A). Data on the densitometric analysis of beclin- $1 / \beta$-tubulin ratio are presented as mean $\pm \mathrm{SD}\left(\mathrm{n}=3 ;{ }^{*} \mathrm{P}<0.05\right.$ and $\left.{ }^{* *} \mathrm{P}<0.01\right)$ (B) Control (100\%); compared to conditions without Ang II.

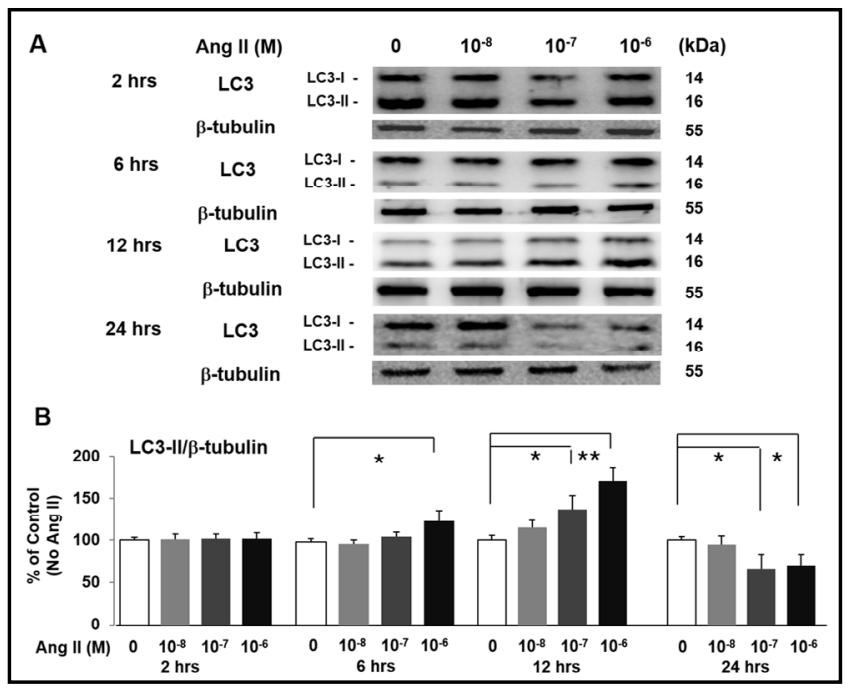

Fig. 2. Effects of Ang II on LC3-II expression assayed using western blot. LC3-II expression is increased by Ang II compared with the control group in time- and concentration-dependent manners (A). At 12 hours of incubation, Ang II increases LC3II expression in a concentration-dependent manner after controlled by $\beta$-tubulin (B). Data on the densitometric analysis are presented as mean $\pm \mathrm{SD}\left(\mathrm{n}=3 ;{ }^{*} \mathrm{P}<0.05\right.$ and $\left.{ }^{* *} \mathrm{P}<0.01\right)$. Control (100\%); compared to conditions without Ang II. 


\section{Cellular Physiology Cell Physiol Biochem 2019;53:747-759 \\ \begin{tabular}{ll|l} 
and Biochemistry & DOl: 10.33594/000000170 & $\begin{array}{l}\text { O } 2019 \text { The Author(s). Published by } \\
\text { Published online: } 18 \text { October } 2019\end{array}$ \\
\cline { 2 - 3 } & Cell Physiol Biochem Press GmbH\&Co. KG
\end{tabular} \\ Seong et al.: Protective Role of Autophagy in Angiotensin II-Induced Podocyte \\ Apoptosis}

\section{Ang II up-regulates LC3-II expression of podocytes}

We evaluated the level of autophagy by measuring the expression of microtubuleassociated protein 1A/1B-light chain 3 (LC3). To determine the effect of Ang II on podocyte LC3-II expression, podocytes were treated with various concentrations of Ang II and incubated for different exposure times. Ang II tended to increase LC3-II protein expression in time- and dose-dependent manners (Fig. 2A, B). Notably, the autophagy signals became evident at 12 hours and, thereafter, decreased to below basal levels at 24 hours. The patterns of LC3-II expression in response to different concentrations of Ang II were similar to those beclin 1 expression.

\section{Ang II increases LC3-II puncta in podocytes}

LC3-II binds to the autophagosomal membrane, allowing the use of immunofluorescence staining of LC3 in monitoring autophagy in cells. To verify whether the Ang II is attributed to autophagy induction, we used bafilomycin $\mathrm{A} 1(10 \mathrm{nM})$ and chloroquine (CQ, $0.1 \mathrm{mM})$ to inhibit autolysosomal degradation. We found that bafilomycin A1 and CQ increased LC3 puncta and Ang II further increased the expression of LC3 puncta significantly, suggesting that Ang II further induced autophagy significantly at 12 hours during the inhibition of autolysosomal degradation by bafilomycin A1 and CQ $(n=4)$ (Fig. 3A, B). The immunofluorescence analysis revealed that Ang II treatment increased LC3 puncta in the cytoplasm (Fig. 3C, D). Ang II treatment increased LC3 puncta in a dose-dependent manner at 12 hours $(\mathrm{n}=3)$ (Fig. 3E). We found that LC3 puncta peaked in the early stage of incubation (at 12 hours) with $10^{-6} \mathrm{M}$

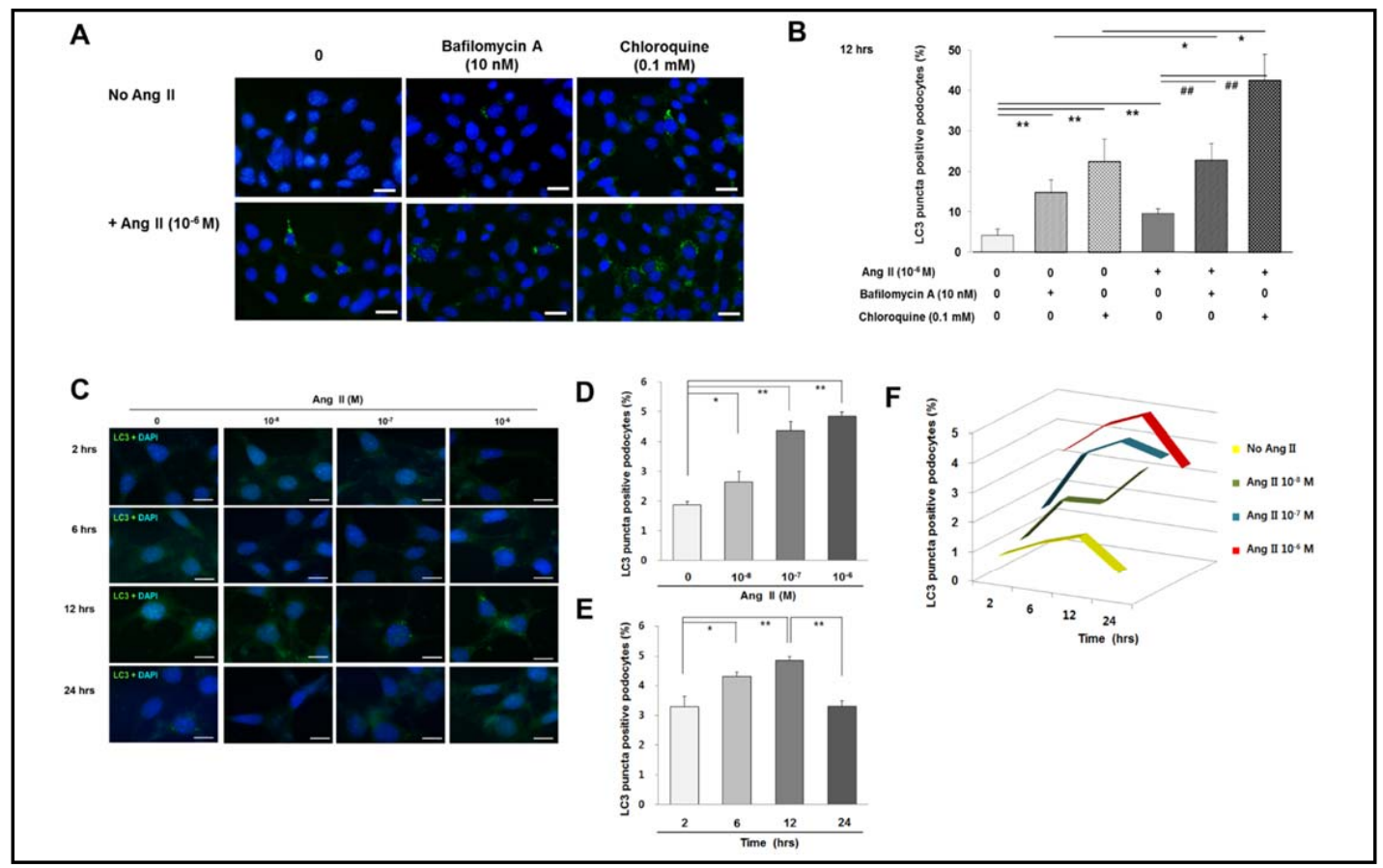

Fig. 3. LC3 puncta in podocytes treated with Ang II. Bafilomycin A1 (10 nM) and chloroquine (CQ, $0.1 \mathrm{mM})$ increased LC3 puncta and additional Ang II further increased the expression of LC3 puncta significantly (A). Magnification, $\times 200$; Bar $=10 \mu \mathrm{m}$. LC3 puncta fluorescence $($ mean \pm SD) were quantified for each experiment $(n=4 ; B)$. At least 30 cells were counted in each individual experiment. Ang II increases LC3 puncta in timeand concentration-dependent manners (C and D). At 12 hours of incubation with Ang II, a concentrationdependent increase in LC3 expression is observed (E). $10^{-6} \mathrm{M}$ Ang II also increases LC3 puncta in a timedependent manner; however, LC3 puncta levels decreased at 24 hours (F). Nuclei are colored in blue by DAPI. Data on the analysis LC3 puncta ratio of podocytes are presented as mean \pm SD ( $n=3$ without notice). Control (100\%); compared to conditions without Ang II. ${ }^{*} \mathrm{P}<0.05$ and ${ }^{* *} \mathrm{P}<0.01$ versus control. ${ }^{\# \#} \mathrm{P}<0.01$ versus condition with Ang II. 


\section{Cellular Physiology Cell Physiol Biochem 2019;53:747-759

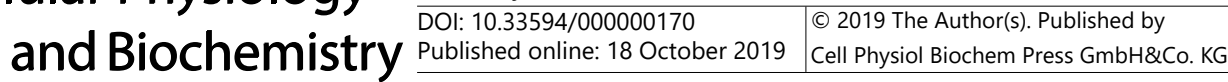 \\ Seong et al.: Protective Role of Autophagy in Angiotensin II-Induced Podocyte \\ Apoptosis}

Ang II; thereafter, LC3 puncta levels dropped to near-basal levels at 24 hours $(n=3)$ (Fig. $3 F$ ). The patterns of LC3 puncta expression in response to different concentrations of Ang II were also similar to the changes of LC3-II and beclin-1 expression data assayed by western blotting.

\section{Ang II promotes podocyte autophagy as detected using TEM}

In the TEM analysis, Ang II treatment impaired the structure of podocytes, showing ultrastructural changes and increased autophagy. A great quantity of independent double membrane structures emerged, gradually extended, and bent. These included autophagosomes containing cytoplasm, lysosomes, and other organelles (Fig. 4A, B). Ang II treatment significantly increased the number of autophagosomes present at 12 hours. In contrast, 3-MA inhibited Ang II-induced autophagosome formation (n = 5) (Fig. 4C).

\section{Ang II induces podocyte apoptosis}

To determine the effect of Ang II on podocyte apoptosis, podocytes were incubated for different exposure times with various concentrations of Ang II. FACS analysis revealed that Ang II treatment increased apoptosis in dose- and time-dependent manners ( $n=3)$ (Fig. 5A, B). TUNEL assays also revealed similar results $(n=3)$ (Fig. $5 C, D)$. Therefore, Ang II induced more apoptosis in podocytes at higher concentrations and longer exposure times. Together, the autophagy and apoptosis data indicate that Ang II induces autophagy before apoptosis in mouse podocytes.

Fig. 4. Effects of Ang II and 3-methyladenine (3-MA) on autophagosomes formation in podocytes detected using transmission electron microscopy. Equal numbers of mouse podocytes were incubated. More autophagosomes were observed in Ang II-treated cells (A, magnification, $\times 3000$; $\operatorname{Bar}=10 \mu \mathrm{m})$. The numbers of autophagosomes (arrows) are increased with Ang II treatment; 3-MA (2 $\mathrm{mM}$ ) attenuates autophagosomes formation at 12 hours (B, magnification, $\times 10000$; $\operatorname{Bar}=1 \mu \mathrm{m}$ ). The numbers of autophagosomes are counted and quantified in 10 random podocytes (C). Data on the analysis of autophagosomes ratio of podocytes are presented as mean \pm SD $(\mathrm{n}=5)$. Control $(100 \%)$; compared to conditions without Ang II. ${ }^{*} \mathrm{P}<0.05$ and ${ }^{* *} \mathrm{P}<0.01$ versus control.

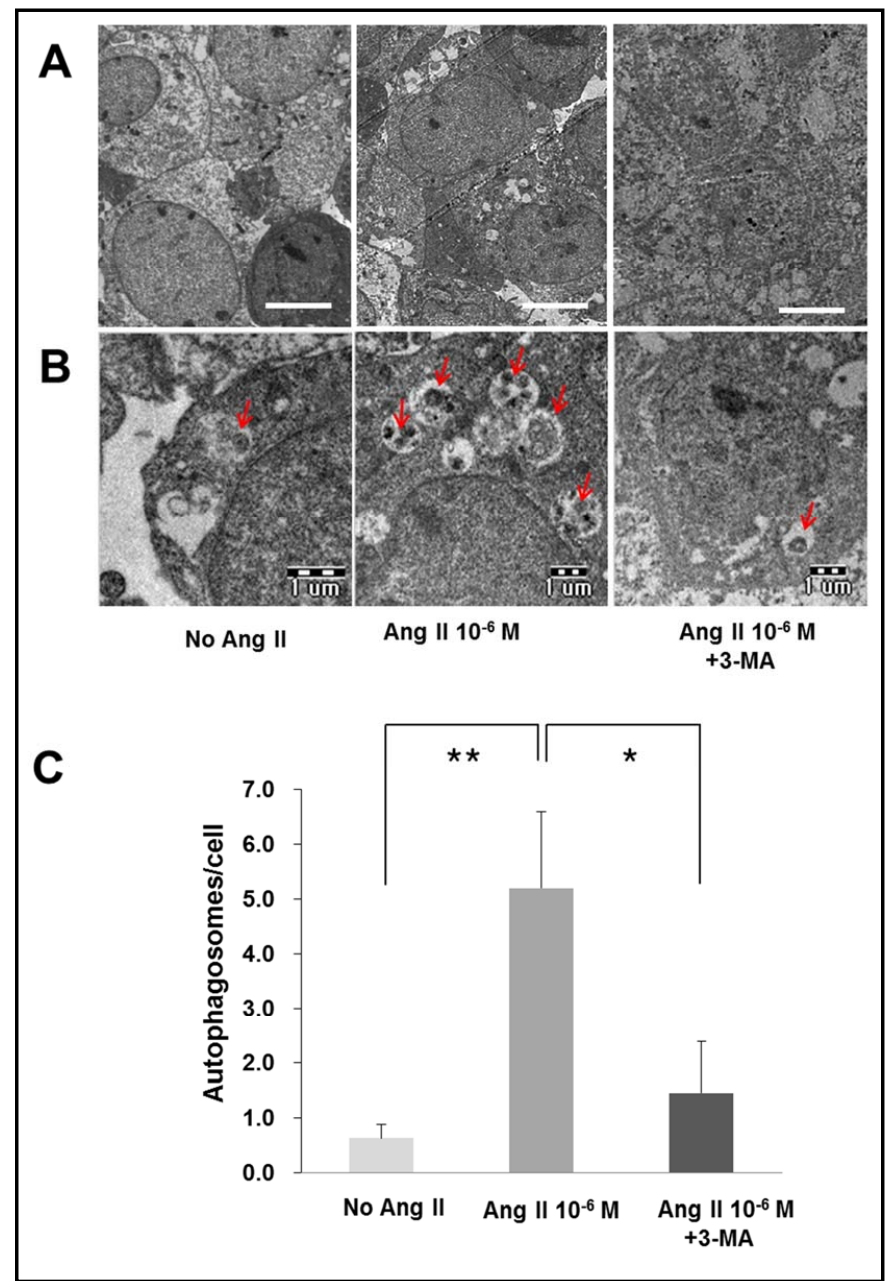




\section{Cellular Physiology Cell Physiol Biochem 2019;53:747-759

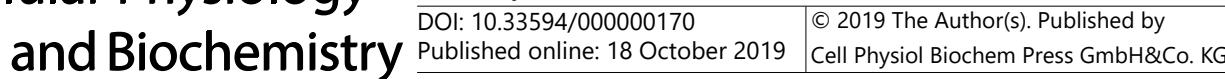 \\ Seong et al.: Protective Role of Autophagy in Angiotensin II-Induced Podocyte \\ Apoptosis}

Inhibitions of PI3-K further induce podocyte apoptosis

Based on our observation that a low percentage of podocytes were apoptotic but a high percentage demonstrated autophagy at 12 hours compared to that at 24 hours after Ang II treatment, we speculated that autophagy may play a cytoprotective role against podocyte apoptosis. To test our hypothesis and investigate the role of PI3-K, two inhibitors were used to suppress autophagy in Ang II-treated podocytes.

Ang II treatment increased LC3 puncta at 12 hours; thereafter, LC3 puncta levels dropped to near-basal levels at 24 hours (Fig. 6A, B), which were similar to the results in Fig. 3D. The PI3-K inhibitors 3-MA (2 mM) and LY294002 (5 $\mu \mathrm{M})$, reduced Ang II-induced LC3 puncta levels; at this time, podocyte apoptosis was significantly increased over that of the control group at 12 and 24 hours (Fig. 6C, D). Thus, PI3-K inhibition further augmented the apoptotic role of Ang II.

Atg5 siRNA inhibits podocyte autophagy and augments Ang II-induced apoptosis

To evaluate the changes of autophagy and apoptosis induced by the suppression of autophagy, we transfected podocytes with Atg5 siRNA. We found that $50 \mathrm{nM}$ of Atg5 siRNA inhibited Atg5 expression by $90 \%$ for 12 hours (Fig. 7A). Atg 5 siRNA reduced LC3 puncta levels, as expected (Fig. 7B). When podocyte autophagy was disrupted by Atg5 siRNA, the number of apoptotic podocytes increased over that observed without or with Ang II treatment in the FACS (Fig. 7C) and TUNEL assays (Fig. 7D). The difference in expression was significant compared with that in the control group.

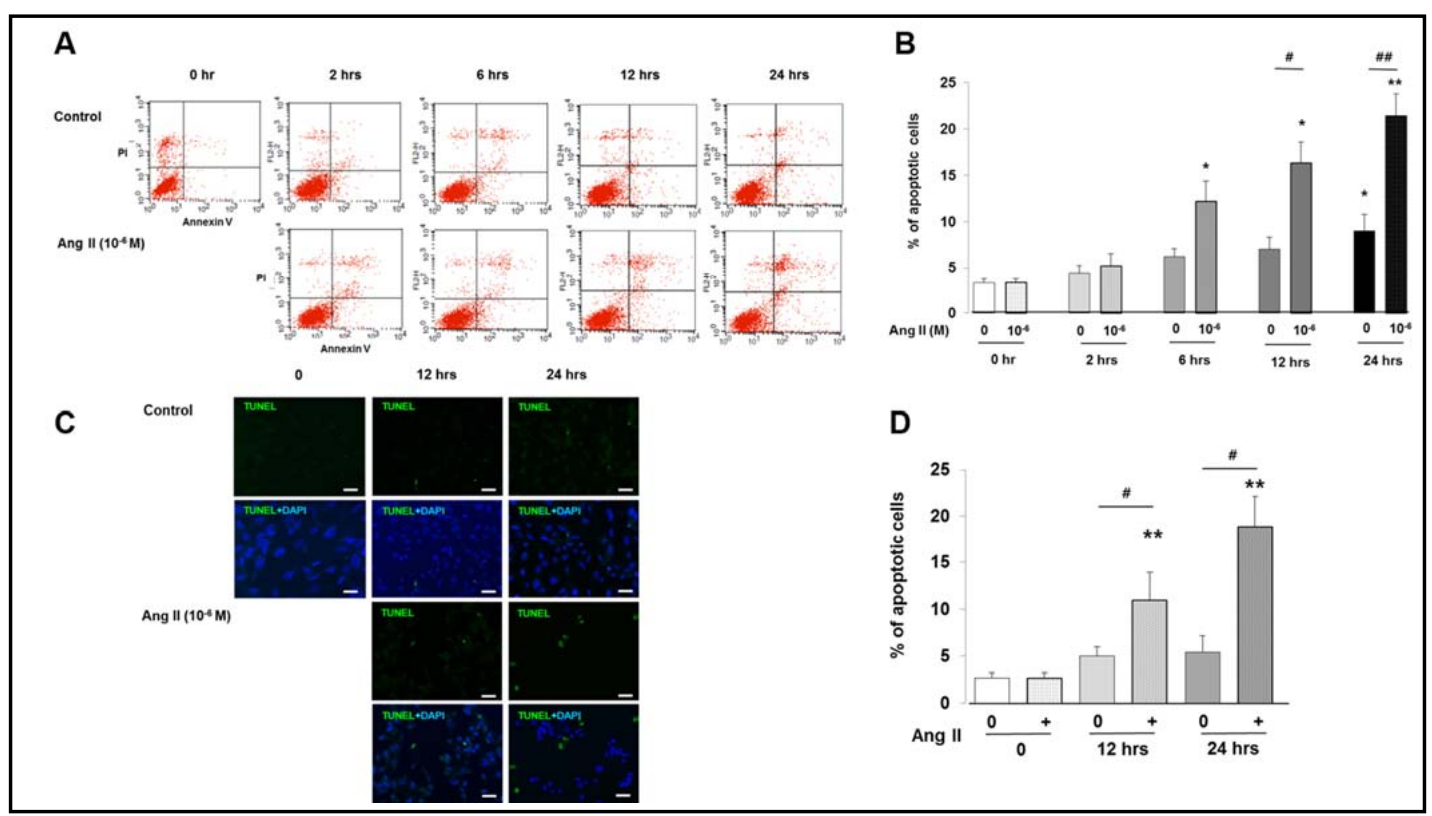

Fig. 5. Ang II induces podocyte apoptosis. Podocyte apoptosis is induced by Ang II in both time- and concentration-dependent manners as determined using FACS (A, B) and TUNEL assay (C, D). Nuclei are colored in blue by DAPI in the TUNEL assay. Data are expressed as mean \pm SD $(n=3) .{ }^{*} \mathrm{P}<0.05$ and ${ }^{* *} \mathrm{P}<0.01$ versus control (without Ang II at 0 hour). ${ }^{\#} \mathrm{P}<0.05$ and ${ }^{\# \#} \mathrm{P}<0.01$ versus control at each incubation time. Magnification, $\times 200$; $\operatorname{Bar}=100 \mu \mathrm{m}$. 


\section{Cellular Physiology

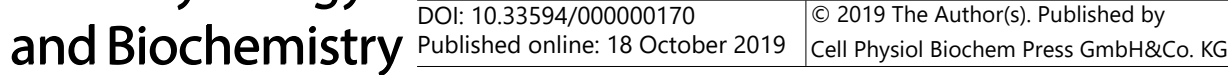 \\ Seong et al.: Protective Role of Autophagy in Angiotensin II-Induced Podocyte Apoptosis}

Fig. 6. Ang II and PI3-K inhibitors induce podocyte apoptosis. Ang II treatment increases LC3 puncta at 12 hours; thereafter, LC3 puncta levels dropped to near-basal levels at 24 hours. The PI3-K inhibitors 3-MA (2 $\mathrm{mM}$ ) and LY294002 (5 $\mu \mathrm{M})$, reduce LC3 puncta levels induced by Ang II (A). The bar chart of the values of LC3 punctapositive cells (B). Ang II induces podocyte apoptosis in a time-dependent manner as determined using FACS assay. Both 3-MA and LY294002 further increase podocyte apoptosis with an additive effect over that of Ang II alone at 12 and 24 hours compared with the control group (C), and representative percentages of apoptotic cells (D). Data are expressed as mean \pm SD $(\mathrm{n}=3) .{ }^{*} \mathrm{P}<0.05$ and ${ }^{* *} \mathrm{P}<0.01$ versus control (without Ang II at each incubation times). ${ }^{\#} \mathrm{P}<0.05$ and ${ }^{\# \# \mathrm{P}<0.01 \text { versus }}$ positive control (with Ang II at each incubation times). Magnification, $\times 200$; $\mathrm{Bar}=10$

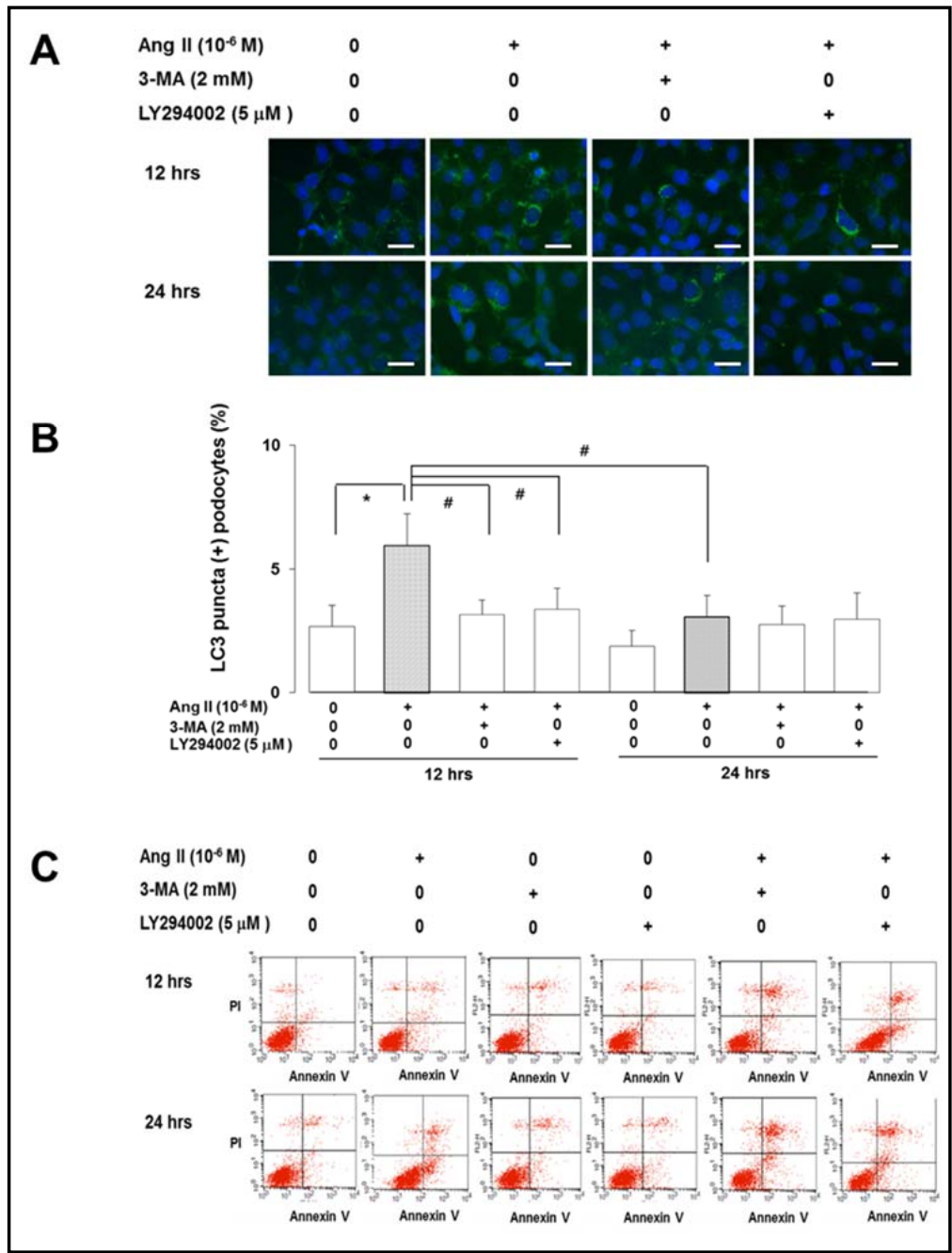
$\mu \mathrm{m}$.

\section{Discussion}

Autophagy and apoptosis are two cellular processes through which injured and aging cells or organelles are eliminated [17-20]. Autophagy is a lysosome-dependent degradation pathway present in most eukaryotic cells and is an important way for the degradation and the recycling of damaged organelles and misfolded proteins for organelle transformation and maintenance cellular homeostasis [17-19]. Autophagy is a mode of stress adaptation that suppresses apoptosis, thereby protecting cells from impending death. Under other conditions, however, excessive autophagy provides an alternative pathway to cell death and is described as autophagic or programmed cell death type II [17-20]. Autophagy occurs at a basal level and stress-induced autophagy primarily serves as an adaptive and protective strategy; on the other hand, apoptosis removes damaged or unwanted cells.

Usually in cell injury, autophagy precedes apoptosis and serves as a line of defense against stress before cellular dismantlement. These cellular events usually occur in cases of autophagy deficiency or in response to prolonged or intense stress [21]. We postulated that the podocyte response following exposure to Ang II might be determined by the duration and concentration of Ang II. This study addresses this statement.

Because the podocyte is a terminally differentiated epithelial cell characterized by a high basal level of autophagy, the absence of podocyte regeneration following cell injury 


\section{Cellular Physiology

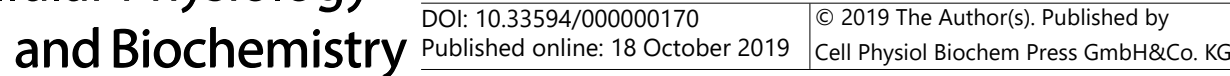 \\ Seong et al.: Protective Role of Autophagy in Angiotensin II-Induced Podocyte \\ Apoptosis}

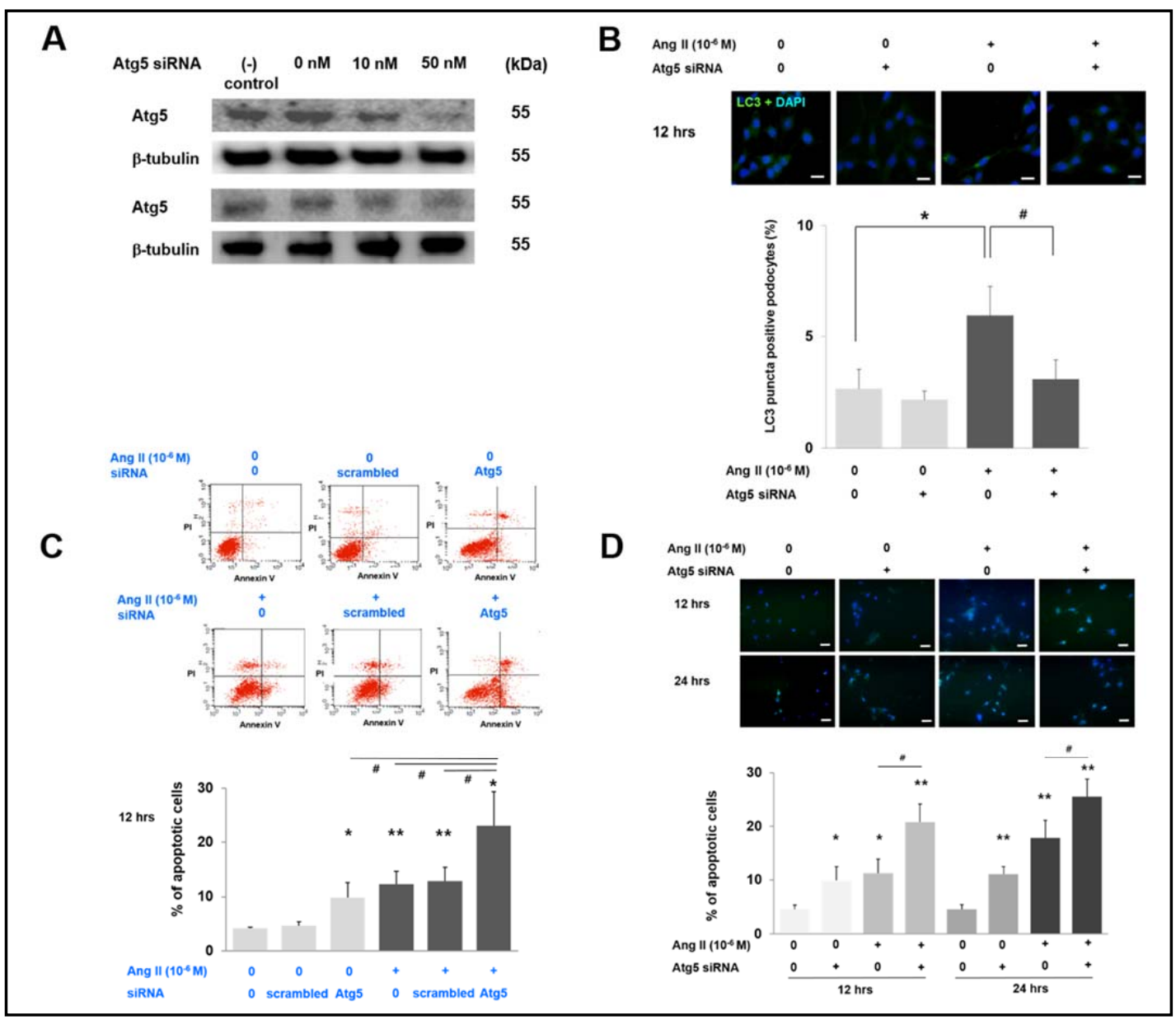

Fig. 7. Ang II induces podocyte apoptosis by modulating Atg5. Transfection of podocytes with Atg5 siRNA significantly inhibits the protein levels of Atg5 (A). Atg5 siRNA (50 nM) reduces LC3 puncta levels increased by $10^{-6} \mathrm{M}$ Ang II at 12 hours (B). When podocyte autophagy is disrupted by Atg5 siRNA, apoptotic podocytes are further increased over that observed with Ang II treatment as determined using FACS (C) and TUNEL assays (D). Nuclei are colored in blue by DAPI in immunofluorescence and TUNEL assay. Magnification, $\times 200$; $\operatorname{Bar}=10 \mu \mathrm{m} .{ }^{*} \mathrm{P}<0.05$ and ${ }^{* *} \mathrm{P}<0.01$ versus control (without Ang II at each incubation times). ${ }^{*} \mathrm{P}<0.05$ versus positive control (with Ang II at each incubation times).

or apoptosis is a major limitation in the approach to glomerular healing. If the injury is tolerable, the podocyte undergoes autophagy to get rid of unwanted or damaged proteins and organelles; otherwise, the injury may tilt the balance toward apoptosis without any attempt for autophagy induction [10-12]. Accordingly, interventions that increase the resistance of this terminally-differentiated cell population to death signals would offer a novel approach to preserve the glomerular filtration barriers [12].

Autophagy is a critical protective mechanism for glomerular homeostatic maintenance in response to various cellular injuries. Recent reports have suggested that autophagy is upregulated in glomerular podocytes and plays a protective role in proteinuric kidney diseases. Hartleben et al. [22] showed that mice lacking Atg 5 in podocytes exhibited strongly increased susceptibility to glomerular disease models, leading to podocyte loss (apoptosis) and age-dependent late-onset glomerulosclerosis. They also found that the basal levels of autophagic flux were high in podocytes, and interruption of this flux by proteasome inhibitors or podocyte injury by puromycin-aminonucleoside (PAN) and adriamycin promoted cytoplasmic accumulation of damaged organelles and protein aggregates. Such damage leads to oxidative stress, endoplasmic reticulum stress (ERS), inflammation and apoptosis, which 
manifested as foot processes effacement and proteinuria. PAN, a podocytotoxic agent, induces autophagy in both murine [11, 23] and human podocytes [24]. LC3-II levels decreased in podocytes damaged by PAN and increase during recovery from the damage in PAN-induced nephrosis animal model and in cultured podocytes [11, 23-25]. In particular, Kang et al. [24] found that autophagy played an early adaptive cytoprotective role to maintain apoptosis at a low level in the early time points; however, as cell stress persisted over time, autophagy was overridden by apoptosis. Autophagy blockade by beclin-1 siRNA, 3-MA, or CQ promoted PAN-induced podocyte apoptosis and damage, as indicated by the loss of podocyte marker proteins, synaptopodin, podocin, and CD2AP [25]. Since both apoptosis and autophagy were triggered by PAN, there may be common signals that involve similar downstream signaling molecules.

Ang II mediates podocyte injury via oxidative stress, ERS, apoptosis, and inflammation, thus, contributing to the development of proteinuria and glomerulosclerosis [3-6]. We found that it also increased podocyte autophagy. The pro-autophagic effect of Ang II was associated with increased podocyte expression of LC3-II and beclin-1. Yadav et al. [12] found that Ang II $\left(10^{-8} \mathrm{M}\right.$, for 36 hours $)$ increased podocyte expression of the autophagic genes, LC3-II and beclin-1; and that Ang II (10-6 M for 24 hours) enhanced podocyte expression of beclin-1. However, our study revealed that Ang II $\left(10^{-6} \mathrm{M}\right)$ increased beclin-1 and LC3-II expression at 6 hours significantly and Ang II $\left(10^{-7}\right.$ and $\left.10^{-6} \mathrm{M}\right)$ increased them further at 12 hours, thereafter, decreased at 24 hours. They also found that rapamycin treatment further increased Ang II-induced autophagy, whereas 3-MA inhibited Ang II-induced autophagy [12]. Shengyou and Li [26] found that Ang II increased podocyte autophagy and induced podocyte apoptosis, which were down-regulated by 3-MA and silencing TRPC6; however, the dose of Ang II and exposure duration were not clearly mentioned in this report. Recently, Wang et al. [27] reported that Ang II induced podocyte autophagy $\left(10^{-7} \mathrm{M}\right.$, for 24 hours) through oxidative stress, at least in part, by regulating the Nox subunit $\mathrm{p} 47$-phox translocation to the membrane. In another recent study, Mao et al. [28] reported that Ang II $\left(10^{-8} \mathrm{M}\right)$ strongly promoted autophagy in immortalized mouse podocyte cells at 24 hours, which effects at low dose $\left(10^{-8} \mathrm{M}\right)$ were similar to ours. They found that Ang II $\left(10^{-8} \mathrm{M}\right)$ at 24 hours increased the activity of AMPK and GSK-3 $\beta$ and downregulated the activity of P70S6K, an mTOR downstream effector, which were compatible with our results on Ang II $\left(10^{-8} \mathrm{M}\right)$ at 24 hours. However, they only did on Ang II $\left(10^{-8} \mathrm{M}\right)$ at 24 hours, but, did not observe sequential or dosedependent changes. As $10^{-8} \mathrm{M}$ of Ang II is not an enough local concentration in glomerulus, their results are not compatible to the generally accepted concept that autophagy is positively regulated by the AMPK activation, but negatively regulated by the mTOR activation. Thus, their experiments did not reflect the pathologic condition induced by Ang II. In our study, we postulated that Ang II-induced podocyte could also be determined by the duration and concentration of Ang II. Our results on autophagic markers, LC-3 and beclin-1, according to the dose of Ang II and exposure duration are similar to the above findings [12, 27]. Together, these results suggest that low doses of Ang II enhance podocyte autophagy over 24 hours, whereas, a higher dose $\left(10^{-6} \mathrm{M}\right)$ enhances podocyte autophagy at an early injury stage of 12 hours and, thereafter, reduces it at 24 hours.

Ang II also induced podocyte apoptosis in vitro in a time-dependent manner and in vivo [12-15]. Since both autophagy and apoptosis may be the outcome of the same insult, it appears that cross-inhibitory balance between apoptosis and autophagy determines the net result [12]. Ang II reportedly enhanced podocyte autophagy and induced podocyte apoptosis, which were down-regulated by silencing TRPC6 and 3-MA [26]. In our study, we found that the inhibition of Ang II-induced autophagy by 3-MA significantly increased podocyte apoptosis. As 3-MA enhances Ang II-induced apoptosis, Ang II-mediated podocyte autophagy might provide partial protection against Ang II-induced apoptosis [12].

Our study results suggest a relationship between autophagy and apoptosis in Ang II-treated mouse podocytes. We found that the expression of LC3-II peaked at 12 hours. Increased LC3 puncta were also observed in the cytoplasm at this time point. Additionally, our study showed that the induction of autophagy was transient in the early stages, and the 


\section{Cellular Physiology Cell Physiol Biochem 2019;53:747-759

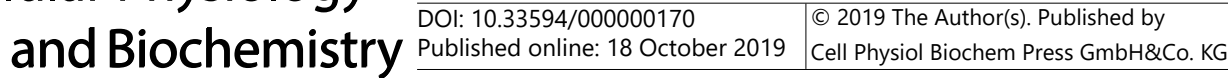 \\ Seong et al.: Protective Role of Autophagy in Angiotensin II-Induced Podocyte \\ Apoptosis}

expression of LC3-II dropped to near basal levels at 24 hours. Combined with our observation that a low percentage of cells was apoptotic in the early stages ( 2 and 6 hours) and apoptosis peaked at later stages (12 and 24 hours), we suggest that Ang II induced stress in podocytes and triggered both autophagy and apoptosis. At the early time point, autophagy played a cytoprotective role to keep apoptosis at a low level; however, as the cell stress persisted over time, the transitional imbalance between autophagy and apoptosis occurred, and autophagy was overwhelmed by apoptosis at later stages. Notably, the cumulative data on autophagy and apoptosis clearly indicate that Ang II induced autophagy before apoptosis in mouse podocytes [24, 25, 29] and cardiomyocytes [30].

In podocyte lipid rafts, CD2AP and nephrin interact with a subunit of class I PI3-kinase, and subsequently stimulate PI3-kinase-dependent activation of the intracellular Akt kinase pathway, which is necessary for the regulation of actin dynamics and cell survival $[9,31]$. PI3-kinase inhibitors, such as, 3-MA, LY294002, and wortmannin, can inhibit both class I and class III PI3-kinase. In particular, while 3-MA persistently blocks the class I PI3-kinase, its suppressive effect on class III PI3-kinase is transient [32]. Because these compounds inhibit the class III PI3-kinase, they block the early stages of autophagosome biogenesis. However, they can also inhibit the class I PI3-kinase, thereby disrupting the Akt pathway and affecting cell viability $[32,33]$. Recently, we found that Ang II-induced podocyte apoptosis is time- and concentration-dependent. LY294002 further increased podocyte apoptosis, which was augmented by CD2AP siRNA but suppressed by AT1R siRNA. These results suggest that Ang II induces podocyte apoptosis by suppressing CD2AP/PI3-kinase signaling [34]. In the present study, we found that PI3-kinase inhibition by 3-MA or LY294002 further increased Ang II-induced podocyte apoptosis and inhibited autophagosome formation.

\section{Conclusion}

In conclusion, the cumulative data of autophagy and apoptosis suggest that Ang II promotes autophagy in mouse podocytes before apoptosis as an early adaptive cytoprotective mechanism for podocyte survival; autophagy is then overwhelmed by apoptosis through a continuous Ang II exposure. In addition, as PI3-kinase interacts with CD2AP and nephrin in podocyte to promote podocyte survival, PI3-kinase inhibition accentuates the imbalance between autophagy and apoptosis causing podocyte injury. The regulation of autophagy has the potential to serve as a novel measure of podocyte injury and proteinuria prevention. Further studies on the pathogenic relationship between autophagy, ERS, and PI3-K, and on upstream signaling, including mTOR-ULK1 and AMPK in Ang II-induced podocyte injury would be needed.

\section{Acknowledgements}

This work was supported by Basic Science Research Program through the National Research Foundation of Korea (NRF) funded by the Ministry of Education (NRF2013R1A1A4A03006207 and 2016R1D1A1B03931888) and partly by Academic-Industrial Cooperative Research Fund of Korean Society of Hypertension (2014).

\section{Disclosure Statement}

The authors have no conflicts of interest to declare. 


\section{Cellular Physiology Cell Physiol Biochem 2019;53:747-759 \begin{tabular}{ll|l}
\hline DOI: 10.33594/000000170 & (c)19 The Author(s). Published by
\end{tabular} and Biochemistry Published online: 18 October 2019 Cell Physiol Biochem Press GmbH\&Co. KG \\ Seong et al.: Protective Role of Autophagy in Angiotensin II-Induced Podocyte \\ Apoptosis}

\section{References}

1 Timmermans PB, Wong PC, Chiu AT, Herblin WF, Benfield P, Carini DJ, Lee RJ, Wexler R, Saye J, Smith R: Angiotensin II receptors and angiotensin II receptor antagonists. Pharmacol Rev 1993;45:205-251.

2 Kobori H, Nangaku M, Navar LG, Nishiyama A: The intrarenal renin-angiotensin system: from physiology to the pathobiology of hypertension and kidney disease. Pharmacol Rev 2007;59:251-287.

3 Aros C, Remuzzi G: The renin-angiotensin system in progression, remission and regression of chronic nephropathies. J Hyperten 2002;20:45-53.

4 Remuzzi G, Benigni A, Remuzzi A: Mechanisms of progression and regression of renal lesions of chronic nephropathies and diabetes. J Clin Investig 2006;116:288-296.

5 Wolf G, Ziyadeh FN: The role of angiotensin II in diabetic nephropathy: emphasis on nonhemodynamic mechanisms. Am J Kidney Dis 1997;29:153-163.

6 Siragy HM, Care RM: Role of the intrarenal renin-angiotensin-aldosterone system in chronic kidney disease. Am J Nephrol 2010;31:541-550.

7 Mundel P, Kriz W: Structure and function of podocytes: an update. Anat Embryol (Berl) 1995;192:385-397.

8 Asanuma K, Mundel P: The role of podocytes in glomerular pathobiology. Clin Exp Nephrol 2003;7:255259.

9 Ha TS: Roles of adaptor proteins in podocyte biology. World J Nephrol 2013;2:1-10.

10 Galluzzi L, Morselli E, Vicencio JM, Kepp 0, Joza N, Tajeddine N, Kroemer G: Life, death and burial: multifaceted impact of autophagy. Biochem Soc Trans 2008;36:786-790.

11 Asanuma K, Tanida I, Shirato I, Ueno T, Takahara H, Nishitani T, Kominami E, Tomino Y: MAP-LC3, a promising autophagosomal marker, is processed during the differentiation and recovery of podocytes from PAN nephrosis. FASEB J 2003;17:1165-1167.

12 Yadav A, Vallabu S, Arora S, Tandon P, Slahan D, Teichberg S, Singhal PC: ANG II promotes autophagy in podocytes. Am J Physiol Cell Physiol 2010;299:C488-C496.

13 Ding G, Reddy K, Kapasi AA, Franki N, Gibbons N, Kasinath BS, Singhal PC: Angiotensin II induces apoptosis in rat glomerular epithelial cells. Am J Physiol Renal Physiol 2002;283:173-180.

14 Jia J, Ding G, Zhu J, Chen C, Liang W, Franki N, Singhal PC: Angiotensin II infusion induces nephrin expression changes and podocyte apoptosis. Am J Nephrol 2008;28:500-507.

15 Wennmann DO, Hsu HH, Pavenstädt H: The renin-angiotensin-aldosterone system in podocytes. Semin Nephrol 2012;32:377-384.

16 Mundel P, Reiser J, Zuniga Mejia Borja A, Pavenstädt H, Davidson GR, Kriz W, Zeller R: Rearrangements of the cytoskeleton and cell contacts induce process formation during differentiation of conditionally immortalized mouse podocyte cell lines. Exp Cell Res 1997;236:248-258.

17 Baehrecke EH: Autophagy: dual roles in life and death? Nat Rev Mol Cell Biol 2005;6:505-510.

18 Gozuacik D, Kimchi A: Autophagy and cell death. Curr Top Dev Biol 2007;78:217-245.

19 Mizushima N, Levine B, Cuervo AM, Klionsky DJ: Autophagy fights disease through cellular self-digestion. Nature 2008;451:1069-1075.

20 Maiuri MC, Zalckvar E, Kimchi A, Kroemer G: Self-eating and self-killing: crosstalk between autophagy and apoptosis. Nat Rev Mol Cell Biol 2007;8:741-752.

21 Fougeray S, Pallet N: Mechanisms and biological functions of autophagy in diseased and ageing kidneys. Nat Rev Nephrol 2015;11:34-45.

22 Hartleben B, Gödel M, Meyer-Schwesinger C, Liu S, Ulrich T, Köbler S, Wiech T, Grahammer F, Arnold SJ, Lindenmeyer MT, Cohen CD, Pavenstädt H, Kerjaschki D, Mizushima N, Shaw AS, Walz G, Huber TB: Autophagy influences glomerular disease susceptibility and maintains podocyte homeostasis in aging mice. Clin Investig 2010;120:1084-1096.

23 Wu L, Feng Z, Cui S, Hou K, Tang L, Zhou J, Cai G, Xie Y, Hong Q, Fu B, Chen X: Rapamycin upregulates autophagy by inhibiting the mTOR-ULK1 pathway, resulting in reduced podocyte injury. PLoS One 2013;8:e63799.

24 Kang YL, Saleem MA, Chan KW, Yung BY, Law HK: The cytoprotective role of autophagy in PAN treated human podocytes. Biochem Biophys Res Commun 2014;443:628-634.

25 Zeng C, Fan Y, Wu J, Shi S, Chen Z, Zhong Y, Zhang C, Zen K, Liu Z: Podocyte autophagic activity plays a protective role in renal injury and delays the progression of podocytopathies. J Pathol 2014;234:203-213. 


\section{Cellular Physiology and Biochemistry}

Cell Physiol Biochem 2019;53:747-759

\begin{tabular}{l|l}
\hline DOI: 10.33594/000000170 & (c) 2019 The Author(s). Published by
\end{tabular}

\begin{tabular}{ll} 
& Cell Physiol Biochem Press GmbH\&Co. KG \\
\hline Seong et al. Protective Role of Autophagy in Angiotensin II-Induced Podocyte
\end{tabular}

Apoptosis

26 Shengyou Y, Li Y. The effects of siRNA-silenced TRPC6 on podocyte autophagy and apoptosis induced by AngII. J Renin Angiotensin Aldosterone Syst 2015;16:1266-1273.

27 Wang W, Cai J, Tang S, Zhang Y, Gao X, Xie L, Mou Z, Wu Y, Wang L, Zhang J: Sinomenine attenuates angiotensin II-induced autophagy via inhibition of $\mathrm{p} 47$-phox translocation to the membrane and influences reactive oxygen species generation in podocytes. Kidney Blood Press Res 2016;41:158-167.

28 Mao N, Tan RZ, Wang SQ, Wei C, Shi XL, Fan JM, Wang L: Ginsenoside Rg1 inhibits angiotensin II-induced podocyte autophagy via AMPK/mTOR/PI3K pathway. Cell Biol Int 2016;40:917-925.

29 Lv Q, Yang F, Chen K, Zhang Y: Autophagy protects podocytes from sublytic complement induced injury. Exp Cell Res 2016;341:132-138.

30 Wang X, Dai Y, Ding Z, Khaidakov M, Mercanti F, Mehta JL: Regulation of autophagy and apoptosis in response to angiotensin II in HL-1 cardiomyocytes. Biochem Biophys Res Commun 2013;440:696-700.

31 Huber TB, Hartleben B, Kim J, Schmidts M, Schermer B, Keil A, Egger L, Lecha RL, Borner C, Pavenstädt H, Shaw AS, Walz G, Benzing T: Nephrin and CD2AP associate with phosphoinositide 3-OH kinase and stimulate AKT-dependent signaling. Mol Cell Biol 2003;23:4917-4928.

32 Wu YT, Tan HL, Shui G, Bauvy C, Huang Q Wenk MR, Ong CN, Codogno P, Shen HM: Dual role of 3-methyladenine in modulation of autophagy via different temporal patterns of inhibition on class I and III phosphoinositide 3-kinase. J Biol Chem 2010;285:10850-10861.

33 Chen Y, Wang BC, Xiao Y: PI3K: a potential therapeutic target for cancer. J Cell Physiol 2012;227:28182821.

34 Park HY, Seong SB, Min SY, Ha TS: CD2-associated protein/phosphoinositide 3-kinase signaling has a preventive role in angiotensin II-induced podocyte apoptosis. Int J Biochem Cell Biol 2016;79:370-381. 